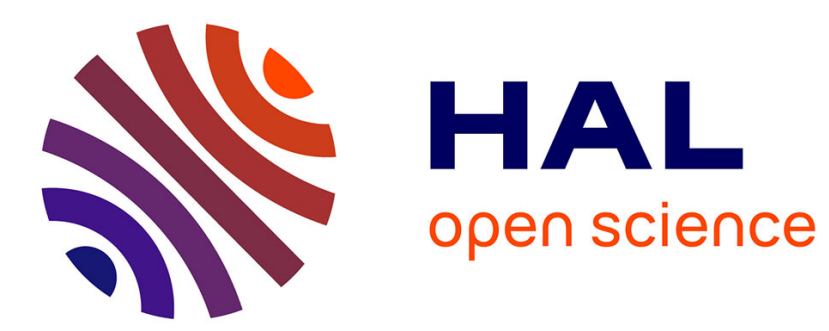

\title{
Une ostéonécrose iatrogène
}

Hélène Geniaux, Sylvain Dumond, Jacques Buxeraud

\section{To cite this version:}

Hélène Geniaux, Sylvain Dumond, Jacques Buxeraud. Une ostéonécrose iatrogène. Actualités Pharmaceutiques, 2019, 58, pp.56 - 57. 10.1016/j.actpha.2019.01.021 . hal-03486099

\section{HAL Id: hal-03486099 \\ https://hal.science/hal-03486099}

Submitted on 20 Dec 2021

HAL is a multi-disciplinary open access archive for the deposit and dissemination of scientific research documents, whether they are published or not. The documents may come from teaching and research institutions in France or abroad, or from public or private research centers.
L'archive ouverte pluridisciplinaire HAL, est destinée au dépôt et à la diffusion de documents scientifiques de niveau recherche, publiés ou non, émanant des établissements d'enseignement et de recherche français ou étrangers, des laboratoires publics ou privés.

\section{다)(1) $(5$}

Distributed under a Creative Commons Attribution - NonCommercial| 4.0 International 
Version of Record: https://www.sciencedirect.com/science/article/pii/S0515370019300503

Manuscript_87a9c4626af06a67f2473e2a0ee0d93c

Dochead pratique

Sous-dochead pharmacovigilance

\section{Une ostéonécrose iatrogène}

\section{Hélène Geniaux ${ }^{\mathrm{a}}$}

Praticien hospitalier

Sylvain Dumond ${ }^{\mathrm{a}}$

Interne en pharmacie

Jacques Buxeraud ${ }^{b^{*}}$

Professeur émérite des Universités

${ }^{\mathrm{a}}$ Centre régional de pharmacovigilance, de pharmaco-épidémiologie et d'information sur les médicaments. Service de pharmacologie, toxicologie et pharmacovigilance, 2 avenue Martin-LutherKing, 87042 Limoges cedex, France

${ }^{\text {b } F a c u l t e ́ ~ d e ~ p h a r m a c i e, ~} 2$ rue du Docteur-Marcland, 87025 Limoges, France

*Auteur correspondant.

Adresse e-mail : jacques.buxeraud@unilim.fr (J. Buxeraud).

\section{Résumé}

L'ostéonécrose est une dégradation anormale et prématurée d'un tissu osseux due à un défaut de vascularisation. La maladie touche principalement des os longs comme le fémur mais aussi des os courts. Il existe de nombreuses causes à cette pathologie parmi lesquelles des ostéonécroses iatrogènes comme celles induites par les bisphosphonates. Un cas de pharmacovigilance avec le bévacizumab utilisé dans un contexte de cancer du sein a été signalé.

(C) 2019

Mots clés - bévacizumab ; iatrogénie ; ostéonécrose ; pharmacovigilance

Summary à venir

(c) 2018

Keywords à venir 
Madame X., 44 ans, atteinte d'un cancer du sein, a commencé un traitement par Avastin ${ }^{\circledR}$ (bévacizumab) et paclitaxel en août 2015.

Depuis fin novembre 2017, elle se plaint d'une douleur au bras gauche, sans facteur déclenchant, localisée au tiers proximal de l'humérus et, de façon concomitante, d'une douleur de l'index gauche. L'épaule est douloureuse, sans aucune amélioration malgré des séances de kinésithérapie.

Le 9 janvier 2018, la patiente est hospitalisée pour sa $57^{\mathrm{e}}{\text { cure } d^{\prime} \text { Avastin }}^{\circledast}$. Elle souffre de plus en plus de son bras et présente une mobilité réduite de l'articulation. À l'imagerie, une rupture du ligament sus-épineux avec début d'ostéonécrose de la tête humérale est observée. Le bévacizumab peut-il être à l'origine de la survenue de cette atteinte?

\section{T1 Le bévacizumab}

TEG1 Commercialisé sous le nom d'Avastin ${ }^{\circledR}$ (solution à diluer pour perfusion), le bévacizumab est indiqué le plus souvent en association dans le cancer colorectal métastatique (CCR) mais également dans d'autres cas tels que le cancer du sein (encadré 1), du poumon non à petites cellules, de l'ovaire et du rein.

TEG1 Le bévacizumab est un anticorps monoclonal ${ }^{1}$ dirigé contre le facteur de croissance endothélial vasculaire (Vascular Endothelial Growth Factor [VEGF]). Il se lie au VEGF facteur clé de la vasculogenèse et de l'angiogenèse, et inhibe donc la liaison du VEGF à ses récepteurs, Flt-1 (VEGFR-1) et KDR (VEGFR-2), à la surface des cellules endothéliales. Ainsi, la neutralisation de l'activité biologique du VEGF :

- fait régresser les vaisseaux tumoraux ;

- normalise les vaisseaux tumoraux restants ;

- inhibe la formation de nouveaux vaisseaux tumoraux.

Ce mécanisme d'action performant permet d'inhiber la croissance tumorale.

\section{Encadré 1. Posologie du bévacizumab dans le cancer du sein}

Perfusion par voie intraveineuse (IV) de $10 \mathrm{mg} / \mathrm{kg}$ de poids corporel une fois toutes les deux semaines ou $15 \mathrm{mg} / \mathrm{kg}$ de poids corporel, une fois toutes les trois semaines.

Poursuivre le traitement jusqu'à la progression de la maladie sous-jacente ou l'apparition d'une toxicité inacceptable.

\section{T1 Les autres inhibiteurs de l'angiogenèse}

D'autres inhibiteurs de l'angiogenèse sont disponibles:

- I'aflibercept (Eyléa ${ }^{\circledR}$, Zaltrap $\left.^{\circledR}\right)$; 
- le ramucirumab $\left(\right.$ Cyramza $\left.^{\circledR}\right)$;

- plusieurs inhibiteurs de la tyrosine kinase (ITK) par voie orale, tels que sunitinib (Sutent ${ }^{\circledR}$ ), sorafénib (Nexavar $\left.{ }^{\circledR}\right)$, pazopanib $\left(\right.$ Votrient $\left.^{\circledR}\right)$, vandétanib (Caprelsa $\left.{ }^{\circledR}\right)$, cabozantinib (Cabométyx ${ }^{\circledR}$ ), axitinib (Inlyta $\left.{ }^{\circledR}\right)$, ponatinib (Iclusig $\left.{ }^{\circledR}\right)$, lenvatinib (Lenvima ${ }^{\circledR}$ ) et régorafénib (Stivarga $\left.{ }^{\circledR}\right)[1,2]$.

\section{T1 Les effets de l'inhibition du VEGF}

\section{TEG1 L'inhibition du VEGF peut avoir des effets :}

- cardiovasculaires (hypertension artérielle, thromboembolie, dysfonction ventriculaire gauche, etc.) ;

- non-cardiovasculaires (protéinurie, saignement, retard de cicatrisation, perforation gastrointestinale, fatigue et dysphonie).

TEG1 D'autres effets rares sont dus à l'inhibition de l'axe du VEGF : leucoencéphalopathie postérieure réversible, hémolyse micro-angiopathique et ostéonécrose [3,4].

\section{T1 L'ostéonécrose sous anti-VEGF}

Les ostéonécroses aseptiques sont liées à l'inhibition de la vascularisation de l'os. D'incidence faible (<1\%), elles prédominent au niveau de la mâchoire [2-5].

\section{La pharmacovigilance}

En France, la pharmacovigilance est assurée par les centres régionaux de pharmacovigilance (CRPV) placés sous la tutelle de l'Agence nationale de sécurité du médicament et des produits de santé (ANSM). Ils ont pour missions de surveiller, évaluer, prévenir les risques médicamenteux potentiels ou avérés et promouvoir le bon usage du médicament. Les CRPV sont au nombre de 31, soit près d'un par centre hospitalier universitaire et forment ainsi, sur le territoire national, un vaste réseau, au plus près des acteurs de santé.

Pour retrouver les coordonnées du CRPV dont l'officine dépende, il convient de consulter la carte interactive (www.rfcrpv.fr/contacter-votre-crpv/) ou le site de I'ANSM (http://ansm.sante.fr/Declarer-un-effet-indesirable/Pharmacovigilance/Centres-regionauxde-pharmacovigilance/(offset)/4).

\section{T2 L'ostéonécrose de la mâchoire}

TEG1 Une étude rétrospective menée chez 3560 patients recevant un traitement contenant du bévacizumab pour un cancer du sein avancé rapporte une ostéonécrose de la mâchoire chez 0,3 à $0,4 \%$ d'entre eux. L'association avec les bisphosphonates augmente d'un facteur 6 le risque ostéonécrotique $[1,4]$. Celui-ci est aussi accru lors de l'association au dénosumab (Prolia ${ }^{\circledR}, \mathbf{X g e v a}^{\circledR}$ ), un anticorps monoclonal (IgG2) humain qui cible le RANKL (receptor activator of nuclear factor 
kappa-B ligand) et qui est indiqué, selon la dose, dans l'ostéoporose (Prolia ${ }^{\circledR}$ ) ou les tumeurs osseuses $\left(\right.$ Xgeva $\left.^{\circledR}\right)$.

TEG1 Les molécules médicamenteuses les plus impliquées dans ces atteintes sont le bévacizumab (Avastin ${ }^{\circledR}$ ) et le sunitinib (Sutent ${ }^{\circledR}$ ) [4]. Cependant, le risque existe avec d'autres ITK qui ciblent le VEGF, tels que sorafenib, axitinib, vandétanib, régorafénib.

\section{T2 Des ostéonécroses des têtes fémorales et humérales}

Divers autres os du squelette peuvent être atteints lors d'un traitement par le bévacizumab, tels que le fémur ou l'humérus :

- une patiente de 72 ans traitée pour un adénocarcinome rectal a développé une ostéonécrose de la tête humérale droite après 15 mois de traitement par bévacizumab à $5 \mathrm{mg} / \mathrm{kg} / 14$ jours en IV associé aux 5-fluorouracile et oxaliplatine $[3,6]$;

- un homme de 65 ans, présentant un adénocarcinome rectal et des métastases pulmonaires, a développé une nécrose avasculaire de la tête fémorale après 11 mois de traitement par bévacizumab IV associé aux 5 -fluorouracile et irinotécan $[3,6]$;

- chez des patients de la population pédiatrique [2-3] pour laquelle la prescription d'Avastin ${ }^{\circledR} \mathrm{n}^{\prime} \mathrm{est}$ pas autorisé, des ostéonécroses non-mandibulaires ont été rapportées.

TEG1 L'incidence des ostéonécroses des têtes fémorales et humérales est estimée par Tessa Tabouret et al. à 4/1 000 chez des patients traités pour un cancer colorectal, ce qui en fait un effet indésirable rare [6]. Les malades observés lors de l'étude avaient une exposition à long terme (> 6 mois) à un anti-VEGF, sans autre facteur de risque prédisposant à l'ostéonécrose, ni traumatisme [6].

\section{T1 Évolution de l'ostéonécrose}

Dans plusieurs cas publiés, l'arrêt du bévacizumab a permis une régression des symptômes et une amélioration de la mobilité et des douleurs [3,6].

\section{T1 Conclusion}

Chez Madame X., l'ostéonécrose est possiblement iatrogène. Au vu des données de la littérature, l'arrêt de ce médicament pourrait permettre une régression des signes. La décision d'arrêt doit être mise en regard des bénéfices attendus.

\section{Points à retenir}

- L'ostéonécrose est une dégradation anormale et prématurée d'un tissu osseux due à un défaut de vascularisation de l'os, qui touche principalement des os longs comme le fémur. 
- La pharmacovigilance a permis de décrire des cas d'ostéonécrose iatrogènes comme celles induites par les bisphosphonates, mais d'autres médicaments sont concernés.

- Le bévacizumab est un anticorps dirigé contre le facteur de croissance endothélial vasculaire (VEGF).

- Le bévacizumab peut induire des effets cardiovasculaires et non-cardiovasculaires. D'autres effets rares, dus à l'inhibition de l'axe du VEGF, incluent un risque d'ostéonécrose.

- L'arrêt du bévacizumab a permis dans plusieurs cas publiés une régression des symptômes et une amélioration de la mobilité et des douleurs.

\section{Note}

${ }^{1}$ Dénomination commune internationale se terminant par "mab" : monoclonal anti body.

\section{Références}

[1] Choueiri TK, Sonpavde G. Toxicity of molecularly targeted antiangiogenic agents: Noncardiovascular effects. 2018. www.uptodate.com/contents/toxicity-of-molecularly-targetedantiangiogenic-agents-non-cardiovascular-effects

[2] Base publique des médicaments. http://base-donnees-publique.medicaments.gouv.fr/

[3] Micromedex ${ }^{\circledR}$ Healthcare Series. 1974-2017. Thomson Micromedex.

[4] www.uptodate.com

[5] Brayfield A. Martindale: The complete drug reference. 38th Revised edition. New York: Pharmaceutical Press; 2014.

[6] Tabouret $\mathrm{T}$, Gregory $\mathrm{T}$, Dhooge $\mathrm{M}$ et al. Long term exposure to antiangiogenic therapy, bevacizumab, induces osteonecrosis. Invest New Drugs. 2015 Oct; 33(5):1144-7

Déclaration de liens d'intérêts

Les auteurs déclarent ne pas avoir de liens d'intérêts. 